\title{
Author's response: BCG and infection with Mycobacterium tuberculosis
}

Thanks to Turner et al for commenting on our article. Turner et al challenge our finding that the Bacillus Calmette-Guérin vaccine (BCG) can prevent Mycobacterium tuberculosis infection (MTI), and propose an alternative explanation: that the absence of a positive interferon gamma release assay (IGRA) could be due to antigenic sin and does not necessarily demonstrate absence of infection.

A positive IGRA is the best available surrogate for MTI, but we are fully aware of its limitations. With the IGRA test, MTI is defined by a T-cell-induced interferon gamma response to $M$ tuberculosis antigens ESAT-6 and CFP-10; a read-out above an established test cut-off reflects MTI. Hence, a read-out below the test cut-off can reflect either no MTI or early containment of MTI, where T-cell responses remain below the cut-off, the latter resulting in measurement error (misclassification). In our study, we observed no difference in MTI vaccine effectiveness when correcting for such misclassification. Additionally, we estimated the association between BCG and MTI with a higher and lower IGRA cut-off than suggested by the manufacturer (cut-off in article $0.35 \mathrm{IU} / \mathrm{mL}$ OR 0.52 (95\% CI 0.32 to 0.85 ), higher cut-off $0.7 \mathrm{IU} / \mathrm{mL}$ OR 0.54 (95\% CI 0.33 to 0.89 ), lower cut-off $0.25 \mathrm{IU} / \mathrm{mL}$ OR 0.50 (95\% CI 0.31 to $0.81)$ ). Both analyses supported the association between BCG and MTI.

The concept of antigenic sin and how this would have a negative influence on a subsequent IGRA test is highly theoretical. In Greenland, the population is virtually naive to non-tuberculous mycobacteria excluding BCG, resulting in minimal cross-reactivity. ${ }^{1}$ Furthermore, ESAT- 6 and CFP-10 are highly immunodominant $M$ tuberculosis antigens, ${ }^{2}$ and no studies have reported a decreased sensitivity of IGRAs in BCG-vaccinated individuals. ${ }^{3}$ Therefore, we find it unlikely that original antigenic sin contributes to the relation between BCG and a negative IGRA. 
We fully agree that more studies on the effect of BCG are needed. Although a series of novel studies suggest that BCG can protect against MTI, there is limited knowledge on the underlying mechanism. ${ }^{4}$ These studies have fuelled an ongoing discussion on whether or not BCG can limit or even prevent bacterial seeding upon $M$ tuberculosis exposure. As an attempt to accelerate the clinical evaluation of novel TB vaccine candidates, Aeras has recently launched a prevention-of-infection trial design in which the ability of BCG to prevent infection will be measured in a randomised, controlled, prospective trial. ${ }^{5}$ Hopefully, these studies will shed further light on the immunological mechanism, which may explain the observational evidence that BCG prevents MTI.

S W Michelsen, ${ }^{1}$ E M Agger, ${ }^{2}$ S T Hoff, ${ }^{2}$ B Soborg, ${ }^{1}$ L Carstensen, ${ }^{1}$ A Koch, ${ }^{1}$ T Lillebaek, ${ }^{3}$ H C F Sorensen, ${ }^{4} \mathrm{~J} \mathrm{Wohlfahrt,}^{1} \mathrm{M}^{\mathrm{M}}$ Melbye ${ }^{1}$

${ }^{1}$ Department of Epidemiology Research, Statens Serum Institut, Copenhagen S, Denmark

${ }^{2}$ Department of Infectious Disease Immunology, Statens Serum Institut, Copenhagen S, Denmark

${ }^{3}$ International Reference Laboratory of

Mycobacteriology, Statens Serum Institut,

Copenhagen S, Denmark
${ }^{4}$ The Tasiilaq District Hospital, Tasiilaq, Greenland

Correspondence to Dr Sascha Wilk Michelsen, Department of Epidemiology Research, Statens Serum Institut, Artillerivej 5, Copenhagen S DK-2300,

Denmark; swm@ssi.dk.

Contributors SWM drafted the manuscript. EMA, STH, BS, MM, JW, LC, AK, TL and HCFS advised on the content. All authors approved the final version. SWM is the guarantor of the manuscript.

Competing interests None.

Ethics approval Commission for Scientific Research in Greenland.

Provenance and peer review Not commissioned; internally peer reviewed.

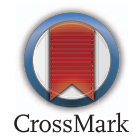

To cite Michelsen SW, Agger EM, Hoff ST, et al. Thorax 2015;70:286-287.

Received 1 September 2014

Accepted 3 September 2014

Published Online First 17 September 2014

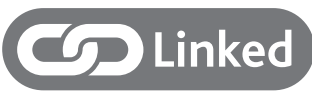

- http://dx.doi.org/10.1136/thoraxjnl-2014-205688

- http://dx.doi.org/10.1136/thoraxjnl-2014-205202

- http://dx.doi.org/10.1136/thoraxjnl-2014-206128
Thorax 2015;70:286-287.

doi:10.1136/thoraxjnl-2014-206258

\section{REFERENCES}

1 Lin MY, Reddy TBK, Arend SM, et al. Cross-reactive immunity to Mycobacterium tuberculosis DosR regulon-encoded antigens in individuals infected with environmental, nontuberculous mycobacteria. Infect Immun 2009;77:5071-9.

2 Skjøt RL, Oettinger T, Rosenkrands I, et al. Comparative evaluation of low-molecular-mass proteins from Mycobacterium tuberculosis identifies members of the ESAT-6 family as immunodominant T-cell antigens. Infect Immun 2000;68:214-20.

3 Pai M, Zwerling A, Menzies D. Systematic review: T-cell - based assays for the diagnosis of latent tuberculosis infection: an update. Ann Intern Med 2008; 149:177-84.

4 Roy A, Eisenhut M, Harris RJ, et al. Effect of BCG vaccination against Mycobacterium tuberculosis infection in children: systematic review and meta-analysis. BMJ 2014;349:g4643.

5 Aeras. Novel Vaccine Trial Design Aims to Answer Key Tuberculosis Questions and Enhance Vaccine Development Strategy-Prevention of infection study underway in South Africa. Press Release March. 2014. http://www.aeras.org/pressreleases/novel-vaccine-trialdesign-aims-to-answer-key-tuberculosis-questions-anden\#.U_r4J3mKBHg (accessed 25 Aug 2014). 